\title{
Possible Existence of Superluminal Photons Inside Microtubules and the Resulting Explanation for Brain Mechanism
}

\author{
Takaaki Musha ${ }^{1,2}$, Luigi Maxmilian Caligiuri ${ }^{2,3}$ \\ ${ }^{1}$ Advanced Science-Technology Research Organization, Yokohama, Japan \\ ${ }^{2}$ Foundation of Physics Research Center (FoPRC), Via Resistenza, Celico (CS), Italy \\ ${ }^{3}$ Faculty of Science, University of Calabria, Arcavacata di Rende (CS), Italy \\ Email Address: \\ takaaki.mushya@gmail.com (T. Musha),max.caligiuri@gmail.com (L. M. Caligiuri)
}

\section{To cite this article:}

Takaaki Musha, Luigi Maxmilian Caligiuri. Possible Existence of Superluminal Photons Inside Microtubules and the Resulting Explanation for Brain Mechanism. American Journal of Optics and Photonics. Vol. 3, No. 5, 2015, pp. 54-57. doi: 10.11648/j.ajop.20150305.11

\begin{abstract}
D.Georgiev presented an idea that consciousness could be the result of quantum computation via short laser-like pulses controlling quantum gates within the brain cortex. However, he later rejected this theory because the wavelength of super radiant photon emission in the infrared spectrum is two orders of magnitude longer than the size of any microtubule cavity. To revive this idea of quantum computation within the brain, the authors propose that the substance within a microtubule cylinder has the characteristics of a metamaterial composed of sub-wavelength structures. Using this hypothesis, we can propose the mechanism for human brain based on superluminal photons.
\end{abstract}

Keywords: Superluminal Photons, Metamaterial, Microtubule, Quantum Computation

\section{Introduction}

S.R. Hameroff suggested in his paper that a centriole cylinder composed of microtubules functions as a waveguide for evanescent photons, which can allow quantum signal processing [1]. Georgiev also proposed the idea that consciousness can be the result of quantum computation via applied laser-like pulses in quantum gates within the brain cortex [2]. Subsequently, Georgiev concluded that this mechanism cannot be used for manipulation of the qubits inside the microtubule cavities, or centrioles, because the photon wavelength is two orders of magnitude longer than the size of these centrioles [3]; super radiant photons in the microtubule cavities could have wavelength of $\lambda=100 \mathrm{~nm}$ or more suggested by Smith [4], incompatible with the length of a moderate-sized microtubule cavity, which is about $1 \mathrm{~nm}$. Therefore, super-radiant emissions could not be used to signal qubits in a fashion similar to standing wave lasers in an ion trap computation. As this mechanism currently stands, the infrared photon cannot account for manipulation of the quantum qubits inside the microtubule cavity. To resolve this problem, we can suggest the hypothesis that the substance in the microtubule cylinder has the characteristics of a metamaterial composed of sub-wavelength structures. If microtubules have similar properties as a metamaterial, evanescent waves inside the microtubule cavity waveguide can propagate below the cutoff frequency, thus we can model the brain mechanism based on a theory of superluminal photons.

\section{Generation Mechanism of Superluminal Photons Inside the Microtubles}

L.M.Caligiuri proposed a novel theoretical model according to which a confined field of faster than light photons field of suitable wavelength can arise from a spontaneous phase transition of the QED quantum vacuum occurring in the water contained inside the microtubules inner volume $[5,6]$. In his papers, it has been shown that, in the water trapped inside microtubules, there exist the conditions for the formation of a macroscopic coherent quantum state in which water molecules oscillate in phase with an electromagnetic (EM) field associated to a suitable electronic transition. Based on the theoretical framework of QED coherence in condensed matter, he shown that water contained inside the hollow volume of brain microtubules is 
able to exhibits a spontaneous superradiant quantum phase transition toward a energetically favoured state, in which the electronic clouds of water molecules coherently oscillate in tune with a self-trapped electromagnetic field within defined space regions (coherent domains).

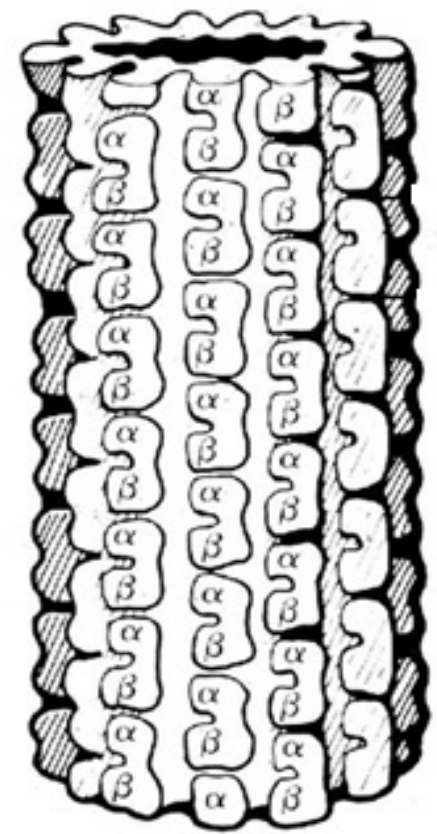

Figure 1. Periodic structure of the microtubule composed of $\alpha$-tubulin and $\beta$-tubulin subunits.

Furthermore, the coherent EM field arising from quantum vacuum oscillating in tune with water molecules is characterized by a negative squared mass of the superradiant photons (since they are trapped inside the coherence domain) and by an "evanescent" tail extending outside the coherence domain itself. In particular, these two latter features allows us to interpret these photons as superluminal evanescent (tunneling) photons that, as it has been also shown in the paper by Caligiuri et al.[5], can be used by living system for recording and retrieving holographycally stored qubits as well as to implement high performance quantum computing inside brain using microtubules substrate as storage material.

\section{Wavelength of the Super Radiant Photons in Microtubules}

Microtubules are a common component of the cytoskeleton, found throughout the cytoplasm. These tubular polymers of tubulin can grow as long as $50 \mu \mathrm{m}$, with an average length of $25 \mu \mathrm{m}$, [7] and are highly dynamic. The outer diameter of a microtubule is about $25 \mathrm{~nm}$ while the inner diameter is about $12 \mathrm{~nm}$. Microtubules are found in all eukaryotic cells and are formed by the polymerization of a dimer of two globular proteins, alpha and beta tubulin. According to the paper by D.D.Georgiev [2], the wavelength of photons of the superradiant emission inside the microtubules within the model developed by Jibu et al. should have

$$
\lambda=h c / E=310 \mu m
$$

by introducing $h=6.63 \times 10^{-34}(\mathrm{~J} \cdot \mathrm{s}), c=3 \times 10^{8}(\mathrm{~m} / \mathrm{c})$ and $E=4 m e V$, where $h$ is Planck's constant, $c$ is light speed and $E$ is the energy difference between the two eigenstates in the superradiating water model [8]. If the super radiant emission is considered in a fashion similar to the use of standing wave lasers in the ion trap computation, there cannot be any nodes and anti-nodes inside the microtubule because the wavelength of superradiant emission is much larger than the size of the microtubule. Differently from the models of quantum optical coherence in cytoskeletal microtuble so far proposed (that considers the energetic transitions of water molecules associated to rotational energy levels of the order of few $m e V$ ), the picture here discussed assumes the coherent system (water + E.M. field) to oscillate in phase with the electronic transition of water from the ground state to the level at energy $E=12.06 \mathrm{eV}$, implying the supperradiant photons, generated inside the coherence domains, to have a wavelength much smaller than the length of a moderately sized microtubule in brain. However the wavelength of such an energy is about $3.75 \times 10^{-8} \mathrm{~m}$ which is larger the diameter of inner radius of microtubles. In this case, the there cannot be superradient transmission of photons inside the microtubule because its size is smaller than the cut-off frequency wavelength. Another possibility that we can propose is that the cylinder formed by the microtubule cavity may contain a medium which has a negative refractive index, similar to a metamaterial [9].

\section{Possible Existence of Metamaterial Inside the Microtubule}

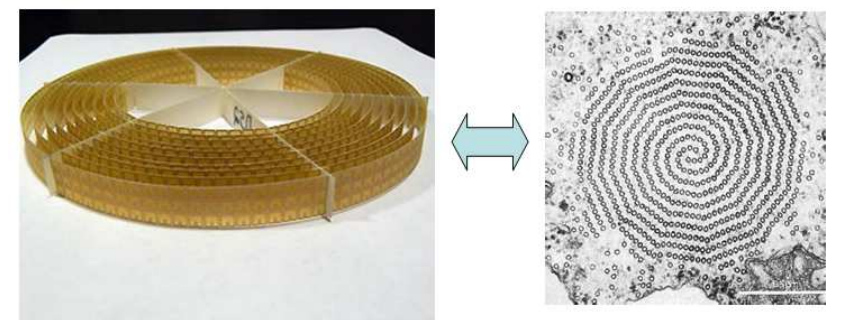

Figure 2. Similarity between the metamaterial (left figure) and the crosssection of an axoneme composed of microtubules (right figure).

Currently metamaterials are artificial materials engineered to have properties that have not been found in nature. They are assemblies of multiple individual elements fashioned from conventional microscopic materials such as metals or plastics, but usually arranged in periodic patterns. Typically, metamaterials have a negative refractive index, a property that is not normally found in nature $[10,11]$. They usually gain their properties from structure rather than composition, using microscopic inhomogeneities to create an effective macroscopic behaviour. Their precise shape, geometry, size, orientation and arrangement can affect the waves of light in an unconventional manner, creating 
material properties which are unachievable with conventional materials. These metamaterials achieve the desired effects by incorporating structural elements of subwavelength sizes, i.e. features that are actually smaller than the wavelength of the waves. Figure. 2 shows the striking similarity between the structures of an electromagnetic metamaterial, constructed of copper slit-ring resonators and wires mounted on interlocking sheets of fiberglass circuit board, and a cross-section of a natural, biological microtubule [12].

As shown in this figure, the cross section of the biological microtubule is very similar to the periodic structure of the artificial electromagnetic metamaterial and we can readily suppose the inner medium of the microtubules cylinder possesses the same metamaterial characteristics. Specifically the characteristic of a negative refractive index, in which the generation of evanescent photons is enhanced, and they can propagate lossless inside the neurons, according to these properties of the metamaterial. From analysis of the amplification of evanescent waves through a rectangular waveguide filled with a metamaterial, of cross section size " $a$ ", J.D.Baena et al. [13] shows the propagation of electromagnetic waves along this wave guide is only possible if $\omega<\omega_{c}$, where $\omega_{c}=(\pi / a) / \sqrt{\varepsilon \mu}$. From this equation, the wavelength for the case where electromagnetic waves propagate through the waveguide becomes

$$
\lambda>2 a
$$

As the average size of microtubules is about $1 \mu \mathrm{m}$ we can see Eq.(2) is satisfied for photons of super radiant emission from the value obtained by Eq.(1). If the microtubules are composed of a metamaterial, the superradiant emission can be used like standing wave lasers in ion trap computation and they can be applied to the manipulation of water qubits inside the microtubule. Therefore it seems highly plausible that macroscopic quantum ordered dynamic systems of evanescent photons in the brain could play an essential role for quantum computations to exist in the brain.

\section{Brain Mechanism from the Standpoint of Superluminal Tunnelling Photons Inside Microtubules}

It has been pointed out, in a paper by Musha [14], evanescent photons generated inside microtubules are superluminal. Ziolkowski also pointed out in his paper, superluminal pulse propagation, permitting consequent superluminal exchange without a violation in causality, is possible in electromagnetic metamaterials [15]. Thus we can suppose microtubule structure permits superluminal propagation of evanescent photons, like the metamaterials, which achieve these properties not from their composition, but from their exactingly, designed structures. Microtubules are hollow structures composed of many identical building blocks, proteins called tubulins, which also make up the internal structure of cilia and flagella. Microtubules provide platforms for intracellular transport and are involved in a variety of cellular processes, including the movement of secretory vesicles, organelles, and intracellular substances. If the inner medium of a microtubules cylinder possesses the characteristics of a metamaterial, with negative refractive index, tunnelling photons will propagate losslessly inside the neuron's microtubules. This will occur in a way which is not restricted by wavelength, and therefore infrared photons could be used for the manipulation of qubits in the brain, allowing the brain to perform quantum computation. The authors have thus proposed the hypothesis quantum computation is conducted within the brain utilizing superluminal photons created inside microtubules [16]. In another paper, by Musha [17], it has been proposed memory within the brain could be due to a holographic mechanism of storing and retrieving superluminal photons in microtubules as follows.

We suppose the cylinder of a microtubule consists of a storage material, whose thickness is much smaller when compared to the penetration depth of an evanescent photon. According to the integral equation for hologram reconstruction, reconstruction can be given by integrating over the volume of the hologram as

$$
U(\vec{r}) \approx \iint U_{0}\left(\vec{r}^{\prime}\right) \exp (-i \beta \vec{n} \cdot \vec{r} /|\vec{r}|) \mathcal{E}(\vec{r}) \cdot \mathrm{d} \vec{r}
$$

where $\mathrm{U}_{0}$ is an illuminating wave, $\mathrm{U}$ is a wave including the information of object wave field and $\beta$ is a parameter satisfying: $\left[\nabla^{2}+\beta^{2}\right] G\left(r^{\prime}, r\right)=-\delta\left(\left|r^{\prime}-r\right|\right)$. This means that the guided readout wave propagates in the same direction as the reference wave during recording as shown in Fig.3. It is diffracted by the hologram structure and the object field can be reconstructed. This is the mechanism of holographic memory of the brain. Utilizing features of both of these mechanisms within the microtubules, we can define how consciousness is created in the human brain.

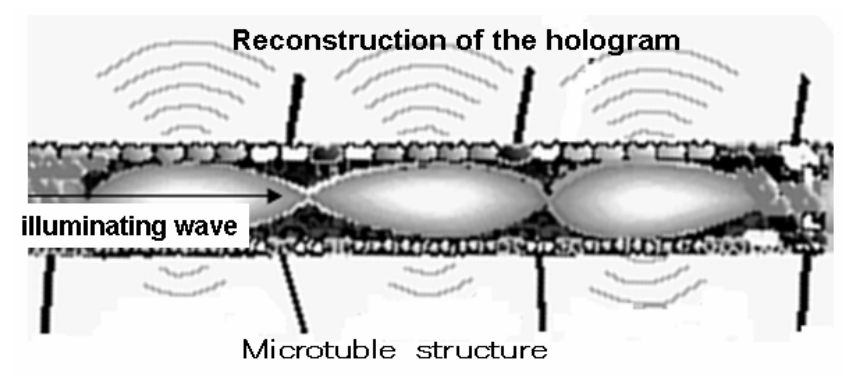

Figure 3. Mechanism of holographic memory by superluminal photons.

According to these suggestions, brain function could be the result of superluminal photons, generated inside the microtubules, which can not only perform quantum computation but also manipulate the storage and retrieval of stored data in the brain, as shown in Fig.4 [18]. 


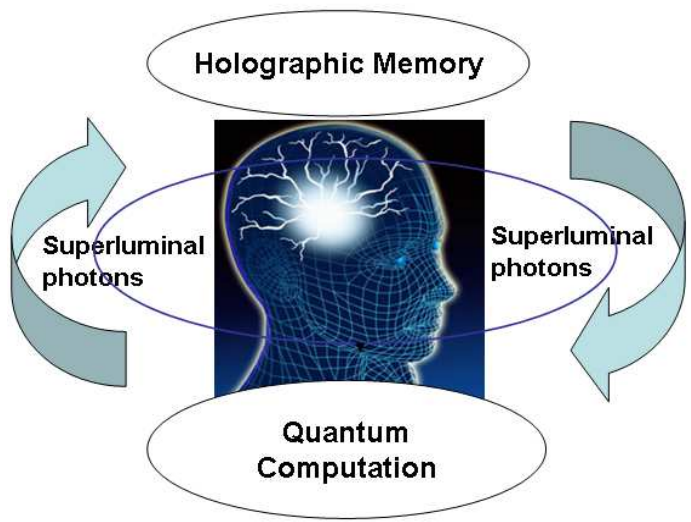

Figure 4. Generation of consciousness via the flow of superluminal photons.

From this figure, we can see that the brain is functioned by superluminal coherent light, which permits the brain for recording and retrieving stored qubits memory holographically as well as to conduct high performance quantum computing inside brain using microtubule structure composed of metamaterials. This may lead to the generation mechanism of consciousness via the flow of superluminal photons inside the human brain. To realize the quantum computer utilizing superluminal photons, we must replace electronic components with optical ones, and an equivalent optical transistor is required. This can be achieved using metamaterial circuits with a non-linear refractive index. Based on this optical computer system like the human brain which utilize metamaterial technology, we have the possibility to realize hypercomputing system in a physical world, which utilizes superluminal photons.

\section{Conclusion}

If the substance inside microtubules has the properties of a metamaterial, we can conclude that superluminal photons generated inside the microtubule could be used for manipulation of qubits, used for a quantum computer system. Thus we can consider the human brain is a quantum computer system utilizing superluminal photons generated inside microtubules.

\section{References}

[1] Hameroff S., Tuszynski J., 2004. Quantum states in proteins and protein assemblies: The essence of life?, SPIE Conference, Grand Canary island.

[2] Georgiev D.D., 2002. Bose-Einstein condensation of tunneling photons in the brain cortex as a mechanism of conscious action, http://cogprint.org/3539/01/tunneling.pdf

[3] Georgiev D., 2004. Quantum computation in the neural microtubles: quantum gates, ordered water and superradiance,arXiv:quant-ph10211080v2.
[4] Smith T., 2003, Quantum consciousness. Water, Light Speed, and $\quad$ Microtubles. http://innerx.net/personal/tsmith/QuanCon2.html.

[5] Caliguiri, L.M., Musha.T,, 2014. Quantum Vacuum Dynamics, Coherence, Superluminal Photons and Hypercomputation in Brain Microtubles, $1^{\text {st }}$ International Conference on Theoretical and Applied Physics (TAP2014), Nov.28-30, 2014, Athens, Greece, 1-11.

[6] Caligiuri L.M., 2015. Zero-Point Field, QED Coherence, Living Systems and Biophotons emission, Open Journal of Biophysics. DOI: 10.4236/ojbiphy.2015.51002.

[7] Satinover J., 2001. The Quantum Brain; The Search for Freedom and the Next Generation of Man, John Wiley \& Sons, Inc.,New York.

[8] Mavromatos N., Nanopoulos D., 1998. Quantum Mechanics in Cell Microtubles: Wild Imagination or Realistic Possibility?,

http://www.theory.caltec.edu/ carlosm/publ/anyonAMStalk.p df.

[9] Mendhe S.E, Icosta Y.R., 2011. Metamaterial Properties and Applications, International Journal of Information Technology and Knowledge Management, Vol.4, No.1, 85-89.

[10] Engheta N., Ziolkowski R.W., 2006. Metamaterial: Physics and Engineering Explorations (Google eBook), John Wiley \& Sons, New York.

[11] Engheta N., Ziolkowski R.W., 2006. Electromagnetic Metamaterial: Physics and Engineering Explorations, WileyIEEE Press.

[12] Pendry J.B., Schurig D., Smith D.R.,2006. Controlling Electromagnetic Fields, Science 312(5514), 1780-1782.

[13] Baena, J.D., Jelinek L., Marques R and Medina F., 2005. Near-perfect tunneling and amplification of evanescent electromagnetic waves in a waveguide filled by a metamaterial: Theory and experiments, Physical Review B.72 075116-1-8.

[14] Musha T., 2005. Superluminal Effect for Quantum Computation that Utilizes Tunneling Photons, Physics Essays, Vol.18, No.4, 525-529.

[15] Ziolkowski R.W., 2001. Superluminal transmission of information through an electromagnetic matamaterial, Physics Review E, Vol.63, 046604-1-13.

[16] Musha T., 2009. Possibility of high performance quantum computation by superluminal evanescent photons in living systems, BioSystems, 96, 242-245.

[17] Musha, T., 2012. Holographic View of the Brain Memory Mechanism Based on Evanescent Superluminal Photons, Information 3,344-350.

[18] Musha T., 2013. Superluminal Particles and Hypercomputation, Lambert Academic Publishing, Saarbrucken, Germany. 